\title{
Acute Ischaemic Enterocolitis Due to a Thrombotic Event in a COVID-19 Patient
}

\section{Montse Adell Trape ${ }^{1 *}$, Anna Curell Garcia ${ }^{1}$, Xavier Guri Azogue ${ }^{2}$, Adriana Carolina Zucchiatti Llanos ${ }^{3}$, Francesc Xavier Nuvials Casals ${ }^{4}$ and Elizabeth Pando ${ }^{5}$}

${ }^{\prime}$ Department of General Surgery, Hospital Universitari Vall d'Hebron, Barcelona, Spain

${ }^{2}$ Department of Radiology, Hospital Universitari Vall d'Hebron, Barcelona, Spain

${ }^{3}$ Department of Pathology, Hospital Universitari Vall d'Hebron, Barcelona, Spain

${ }^{4}$ Department of Intensive Care Medicine, Hospital Universitari Vall d'Hebron, Barcelona, Spain

${ }^{5}$ Department of Hepato-Biliary-Pancreatic and Transplant Surgery, Hospital Universitari Vall d'Hebron, Barcelona, Spain

\author{
A RTICLE INFO \\ Article history: \\ Received: 28 December, 2020 \\ Accepted: 13 January, 2021 \\ Published: 25 January, 2021 \\ Keywords: \\ COVID-19 \\ thrombotic event \\ ischaemic enterocolitis \\ urgent exploratory laparotomy
}

\begin{abstract}
A B S T R A C T
We describe the case of a COVID-19 patient who developed an intestinal ischaemia with thrombotic origin, confirmed by anatomo-pathological examination. A middle-aged woman affected with COVID-19 was evaluated in March 2020. Reverse transcriptase-polymerase chain reaction (RT-PCR) to detect SARS-CoV2 was performed. On the 16th day of being hospitalized, she presented with hemodynamic and respiratory impairment. The physical examination revealed abdominal distension. An urgent abdominal CT scan reported signs of pneumatosis in the distal ileum and ascending colon suggestive of ischaemia (Figure 1D), with mural irregularity predominantly in the ileum. An urgent exploratory laparotomy was performed. Ischaemia of the right colon, as well as patched ischaemia of the distal and middle ileum, was found (Figures 2A \& 2B). A right hemicolectomy and resection of the distal-middle ileum with a terminal ileostomy were performed. Pathological examination reported: severe ischaemic colitis, with areas of mucosal necrosis and the presence of abundant small-calibre thrombi in the submucosa and medium-caliber vessels of the mesocolon. Re-examination of the CT scan revealed findings consistent with right lower lobe segmental pulmonary embolism, as well as inferior vena cava filling defects consistent with thrombi. COVID-19 is associated with a large and misleading field of complications, including coagulopathy. The combination of viral injury, cytokine release, and damage-associated molecular patterns induce localized microvascular inflammation, which triggers endothelial activation, leading to vasodilation and prothrombotic conditions. We report this case to alert physicians that they should be vigilant for signs of abdominal thromboembolic complications in patients suffering from COVID-19.
\end{abstract}

\section{Introduction}

We describe the case of a COVID-19 patient, who developed an intestinal ischaemia with thrombotic origin, confirmed by anatomopathological examination.

\section{Methods}

A middle-aged woman affected with COVID-19 was evaluated at Vall d'Hebron University Hospital in March, 2020. Reverse transcriptase- polymerase chain reaction (RT-PCR) to detect SARS-CoV-2 was performed. Information was obtained from medical records.

\section{Results}

A 57-year-old woman, ex-smoker, with a history of GOLD-I chronic obstructive pulmonary disease, presented with a 7-day productive cough, chest pain and dyspnea. The oropharyngeal swab for SARS-CoV-2 was positive. Lab tests at admission were: D-Dimer $1103 \mathrm{ng} / \mathrm{mL}$, IL-6 47.6, C-reactive protein (CRP) $15.7 \mathrm{mg} / \mathrm{dL}$, Ferritin $254 \mathrm{ng} / \mathrm{mL}$. Arterial blood

\footnotetext{
${ }^{*}$ Correspondence to: Montse Adell Trapé, Department of General Surgery, Hospital Universitari Vall d'Hebron, Barcelona, Spain; E-mail: montse.adellt@gmail.com

(C) 2021 Montse Adell Trapé. This is an open-access article distributed under the terms of the Creative Commons Attribution License, which permits unrestricted use, distribution, and reproduction in any medium, provided the original author and source are credited. Hosting by Science Repository. http://dx.doi.org/10.31487/j.SCR.2021.01.14
} 
gas showed severe hypoxemia $(\mathrm{PaFiO} 2=58 \mathrm{mmHg})$, and chest $\mathrm{X}$-ray demonstrated bilobar pulmonary infiltrates. The treatment prescribed was: Ceftriaxone, Azithromycin, Lopinavir/Ritonavir, low-molecularweight heparin $(0,5 \mathrm{mg} / \mathrm{Kg})$ and high flow oxygen therapy. Despite this, her clinical condition deteriorated. A dose of beta-interferon was given, $(16 \mathrm{UM})$ and she was transferred to the ICU. She required orotracheal intubation and prone positioning at days 2, 4 and, 6. She developed a persistent multi-organ failure, requiring noradrenaline (maximal dose: $0,9 \mu \mathrm{g} / \mathrm{Kg} / \mathrm{min}$ ) and renal replacement therapy on day 8 . She was treated for a catheter-related bloodstream infection due to C. albicans and tracheobronchitis due to $S$. aureus. During the two weeks of ICU admission, the respiratory condition progressively improved, decreasing the oxygen $\mathrm{FiO} 2$ (from 0.5 to 0.3 ) and PEEP (from 12 to $6 \mathrm{~cm} \mathrm{H}_{2} \mathrm{O}$ ). On the 16th day, she presented with hemodynamic and respiratory impairment. The physical examination revealed abdominal distension. Lab tests showed: severe metabolic acidosis ( $\mathrm{pH}, 6.68)$, leukocytosis of 13000, alanine transaminase $183 \mathrm{U} / \mathrm{l}$, and CRP $25.89 \mathrm{mg} / \mathrm{dL}$. An urgent thoracoabdominal CT scan reported signs of pneumatosis in the distal ileum and ascending colon suggestive of ischaemia (Figure 1D), with mural irregularity predominantly in the ileum, together with bilateral and multilobar ground glass opacities consistent with the already known COVID-19 infection (Figure 1A).
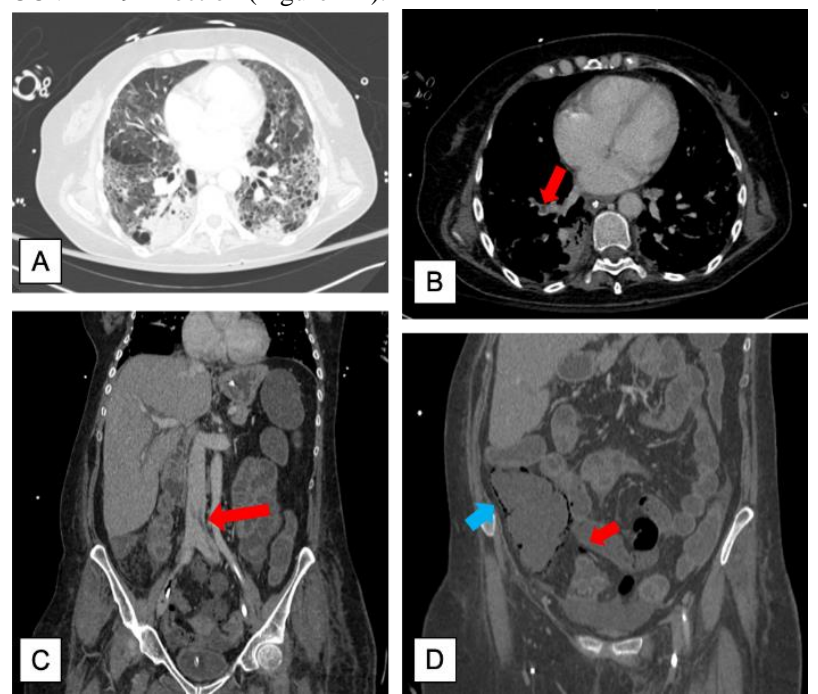

Figure 1: A) Chest CT scan showing an emphysematous lung with COVID-19 infection: bilateral and multilobar ground-glass opacities with a peripheral distribution, mainly in the lower lobes. B) Filling defect within the pulmonary vasculature in the right lower lobe compatible with pulmonary embolism. C) Filling defect in the inferior vena cava due to a thrombus. D) Intramural gas of the cecum (blue arrow) and mesenteric vein gas (red arrow) caused by mesenteric ischaemia.

An urgent exploratory laparotomy was performed. Ischaemia of the entire right colon, as well as patched ischaemia of the distal-middle ileum, were found (Figures 2A \& 2B). A right hemicolectomy and resection of the distal-middle ileum with a terminal ileostomy were performed. During the following hours, the patient deteriorated her multiorgan failure status, which finally resulted in her death. Pathological examination reported: severe ischaemic colitis, with areas of mucosal necrosis and the presence of abundant small-caliber microthrombi in the submucosa and medium-caliber vessels of the mesocolon (Figure 2C), together with moderate inflammatory infiltrates. Re-examination of the CT scan revealed findings consistent with right lower lobe segmental pulmonary embolism (Figure 1B), as well as inferior vena cava filling defects consistent with thrombi (Figure 1C).

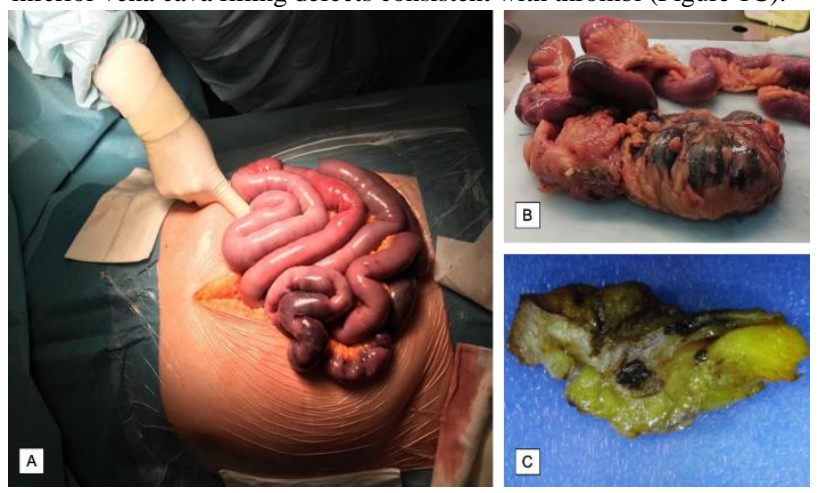

Figure 2: A) Intraoperative image of the open exploratory laparotomy showing segmentary ischaemia of the distal and middle ileum. B) Surgical specimen including the right hemicolectomy and resection of the distal and middle ileum with signs of ischaemic colitis (necrotic plaques on its wall and areas of mucosal necrosis). C) Mesocolon specimen with abundant small-caliber microthrombi in the submucosa and medium-caliber vessels.

\section{Discussion}

COVID-19 is associated with a large and misleading field of complications, including coagulopathy [1]. Emerging data suggests that severe COVID-19 reflects a confluence of vascular dysfunction, thrombosis, and dysregulated inflammation [2]. Both D-Dimer and antiphospholipid antibodies can increase transiently in critically ill patients with COVID-19 [3]. The combination of viral injury, cytokine release, and damage-associated molecular patterns induce localized microvascular inflammation, which triggers endothelial activation, leading to vasodilation and prothrombotic conditions [2]. This could lead to both venous and arterial thromboembolic events [3].

Thromboembolic complications occur in more than $20 \%$ of hospitalized patients with COVID-19, and this percentage rises to $31 \%$ among ICU patients [4]. A recent study demonstrates that CT pulmonary angiography and/or ultrasonography confirmed venous thromboembolism in $27 \%$ of ICU patients [5]. Moreover, radiological findings consistent with cerebral ischaemic stroke have been reported in COVID-19 patients [6]. We report this case of an acute enterocolitis ischaemia with a thrombotic origin in a patient affected with SARSCoV-2, to alert physicians that they should be vigilant for signs of abdominal thromboembolic complications, and also search actively for these complications ordering diagnostic tests when this is suspected.

\section{REFERENCES}

1. Zhang Y, Cao W, Xiao M, Li YJ, Yang Y et al. (2020) Clinical and coagulation characteristics of 7 patients with critical COVID-2019 pneumonia and acro-ischemia. Zhonghua Хие Ye Xиe Za Zhi 41: E006. [Crossref]

2. Leisman DE, Deutschman CS, Legrand M (2020) Facing COVID-19 in the ICU: vascular dysfunction, thrombosis, and dysregulated inflammation. Intensive Care Med 46: 1105-1108. [Crossref] 
3. Zhang Y, Xiao M, Zhang S, Xia P, Cao W et al. (2020) Coagulopathy and Antiphospholipid Antibodies in Patients with Covid-19. N Engl J Med 382: e38. [Crossref]

4. Lodigiani C, Iapichino G, Carenzo L, Cecconi M, Ferrazzi P et al. (2020) Venous and arterial thromboembolic complications in COVID19 patients admitted to an academic hospital in Milan, Italy. Thromb Res 191: 9-14. [Crossref]
5. Klok FA, Kruip MJHA, van der Meer NJM, Arbous MS, Gommers DAMPJ et al. (2020) Incidence of thrombotic complications in critically ill ICU patients with COVID-19. Thromb Res 191: 145-147. [Crossref]

6. Helms J, Kremer S, Merdji H, Clere Jehl R, Schenck M et al. (2020) Neurologic Features in Severe SARS-CoV-2 Infection. $N$ Engl J Med. 382: 2268-2270. [Crossref] 